Research Article

\title{
Benign Effect of Extremely Low-Frequency Electromagnetic Field on Brain Plasticity Assessed by Nitric Oxide Metabolism during Poststroke Rehabilitation
}

\author{
Natalia Cichoń, ${ }^{1}$ Piotr Czarny, ${ }^{2}$ Michał Bijak, ${ }^{1}$ Elżbieta Miller, ${ }^{3,4}$ Tomasz Śliwiński, ${ }^{5}$ \\ Janusz Szemraj, ${ }^{2}$ and Joanna Saluk-Bijak ${ }^{1}$ \\ ${ }^{1}$ Department of General Biochemistry, Faculty of Biology and Environmental Protection, University of Lodz, Pomorska 141/143, \\ Lodz, Poland \\ ${ }^{2}$ Department of Medical Biochemistry, Medical University of Lodz, Mazowiecka 6/8, Lodz, Poland \\ ${ }^{3}$ Department of Physical Medicine, Medical University of Lodz, Pl. Hallera 1, Lodz, Poland \\ ${ }^{4}$ Neurorehabilitation Ward, III General Hospital in Lodz, Milionowa 14, Lodz, Poland \\ ${ }^{5}$ Department of Molecular Genetics, Laboratory of Medical Genetics, Faculty of Biology and Environmental Protection, \\ University of Lodz, Lodz, Pomorska 141/143, Lodz, Poland \\ Correspondence should be addressed to Natalia Cichoń; natalia.cichon@biol.uni.lodz.pl
}

Received 12 May 2017; Revised 2 July 2017; Accepted 14 August 2017; Published 12 September 2017

Academic Editor: Tanea T. Reed

Copyright (c) 2017 Natalia Cichoń et al. This is an open access article distributed under the Creative Commons Attribution License, which permits unrestricted use, distribution, and reproduction in any medium, provided the original work is properly cited.

\begin{abstract}
Nitric oxide (NO) is one of the most important signal molecules, involved in both physiological and pathological processes. As a neurotransmitter in the central nervous system, NO regulates cerebral blood flow, neurogenesis, and synaptic plasticity. The aim of our study was to investigate the effect of the extremely low-frequency electromagnetic field (ELF-EMF) on generation and metabolism of $\mathrm{NO}$, as a neurotransmitter, in the rehabilitation of poststroke patients. Forty-eight patients were divided into two groups: ELF-EMF and non-ELF-EMF. Both groups underwent the same 4-week rehabilitation program. Additionally, the ELF-EMF group was exposed to an extremely low-frequency electromagnetic field of $40 \mathrm{~Hz}, 7 \mathrm{mT}$, for 15 min/day. Levels of 3-nitrotyrosine, nitrate/nitrite, and TNF $\alpha$ in plasma samples were measured, and NOS2 expression was determined in whole blood samples. Functional status was evaluated before and after a series of treatments, using the Activity Daily Living, Geriatric Depression Scale, and Mini-Mental State Examination. We observed that application of ELF-EMF significantly increased 3-nitrotyrosine and nitrate/nitrite levels, while expression of NOS2 was insignificantly decreased in both groups. The results also show that ELF-EMF treatments improved functional and mental status. We conclude that ELF-EMF therapy is capable of promoting recovery in poststroke patients.
\end{abstract}

\section{Introduction}

Cardiovascular diseases, including ischemic stroke (IS), are a serious problem of the modern age, killing 4 million people each year in Europe [1]. Stroke is caused by ischemia of brain tissue. Brain structure damage occurring during ischemia/ reperfusion is due to the generation of significant amounts of reactive oxygen species and inflammatory mediators [2]. Damage to brain tissue as a result of a stroke cannot be undone. However, the most important part of poststroke therapy is immediate and long-term rehabilitation, considering the enormous plasticity of the brain [3]. Although extremely low-frequency electromagnetic field (ELF-EMF) therapy is not a standard treatment in the poststroke rehabilitation, some authors suggest its increased positive effect on patients [4]. ELF-EMF treatment is based on regeneration, osteogenesis, analgesics, and anti-inflammatory action. Its biological effect is related to processes of ion transport, cell 
proliferation, apoptosis, protein synthesis, and changes in the transmission of cellular signals [5]. The regenerative and cytoprotective effect of ELF-EMF is based on mechanism associated with nitric oxide induction, collateral blood flow, opioids, and heat shock proteins [6].

Nitric oxide (NO) is an unstable, colourless, water-soluble gas with a short half-life $(3-6 \mathrm{sec})$. The compound has one unpaired electron, which makes it a highly reactive free radical. It is characterized by the multiplicity of action in the body, in both physiological and pathological conditions [7]. Synthesis of NO in the organism is catalysed by nitric oxide synthase (NOS), occurring in three isoforms: neuronal (nNOS), inducible (iNOS), and endothelial (eNOS), encoded by different genes whose expression is subject to varying regulation. The constituent isoforms of NOS are eNOS and nNOS, whose activity is associated with concentration of calcium ions and the level of calmodulin in a cell, as well as with hypoxia, physical activity, and the level of certain hormones, that is, oestrogens [8]. In contrast, because it is closely related with the calmodulin, iNOS does not require a high concentration of calcium ions but is regulated by various endogenous and exogenous proinflammatory factors [9].

The two-stage synthesis of NO consists of the oxidation of L-arginine to $\mathrm{N} \omega$-hydroxy-L-arginine and, under the influence of NOS and oxygen, formation of L-citrulline and release of NO. All isoforms of NOS require the same cofactors: nicotinamide adenine dinucleotide phosphate (NADPH), flavin mononucleotide (FMN), flavin adenine dinucleotide (FAD), tetrahydrobiopterin (BH4), iron protoporphyrin IX (heme), and $\mathrm{O}_{2}$ [7].

Nitric oxide is one of the most important signal molecules, involved in both physiological and pathological processes. One of the major functions of $\mathrm{NO}$ is as a potent vasodilation, increasing the blood flow and regulation of blood pressure, which has been used in clinical practice for many years. Deficiency of this compound is observed in various disorders of many systems: cardiovascular, gastrointestinal, respiratory, and genitourinary [10]. The beneficial effects of NO lie in its platelet inhibition, macrophage cytotoxicity (antibacterial, antiviral, and antiparasitic), and protection of the mucosal lining of the digestive system. On the other hand, excessive expression of iNOS can be disadvantageous, for example, during sepsis. The adverse action of $\mathrm{NO}$ is associated with the production of superoxide anions and subsequent generation of peroxynitrite and hydroxyl radicals, which are highly toxic [11].

In the central nervous system, $\mathrm{NO}$ as a neurotransmitter regulates cerebral blood flow, as well as neurogenesis and synaptic plasticity. Furthermore, neuronal death is caused by high concentrations of NO by caspase-dependent apoptosis process and promotion of inflammation. Elevated levels of nitric oxide promote necrosis by energy depletion. On the basis of these mechanisms, NO is involved in the etiology of many neurological diseases, such as major depression, schizophrenia, epilepsy, anxiety, and drug addiction [12].

Our study was designed to investigate the effect of ELF-EMF on the metabolism of NO, as a signal molecule in the central nervous system, in the rehabilitation of acute poststroke patients.

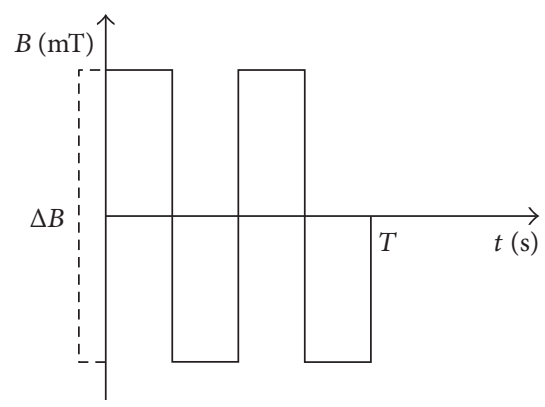

Figure 1: ELF-EMF description. $\Delta B=5 \mathrm{mT} ; T=1.3 \mathrm{sec}$.

\section{Materials and Methods}

2.1. Blood Sample Collection. Blood samples were collected into $\mathrm{CPDA}_{1}$-containing tubes (Sarstedt, Nümbrecht, Germany). Immediately upon doing so, a portion of the sample was frozen at $-80^{\circ} \mathrm{C}$ and the rest of the samples centrifuged to isolate the plasma $(15 \mathrm{~min}, 1500 \mathrm{~g})$ at $25^{\circ} \mathrm{C}$. Blood samples were collected twice, at an interval of 14 days before and after a standard 10 sessions of therapy. For additional analysis of 3-nitrotyrosine levels, the blood samples were collected three times, at an interval of 28 days: before treatment, after 10 treatments, and after 20 treatments. All blood samples were taken in the morning (between $7 \mathrm{am}$ and $9 \mathrm{am}$ ) under patient fasting condition and stored using the same protocol.

\subsection{Subject Presentation. Forty-eight poststroke patients} were enrolled in the study. Participants were randomly divided into two groups: ELF-EMF $(n=25)$ and non-ELFEMF $(n=23)$. Patients with metal and/or electronic implants (pacemakers, etc.) were excluded from the ELF-EMF group, for safety reasons. The ELF-EMF group had already undergone ELF-EMF therapy with specific parameters $(40 \mathrm{~Hz}$ frequency, magnetic induction of $5 \mathrm{mT}(\Delta B)$, rectangular and bipolar waveforms) (Figure 1), which was conducted using a Magnetronic MF10 generator (EiE Elektronika i Elektromedycyna, Otwock, Poland). The parameters were selected on the basis of the fact that low-intensity stimuli improve the vital functions of the body. In addition, rectangular pulses are more intense than sinusoidal and trapezoid, while bipolar pulses show more range of changes than unipolar pulses [13]. The ELF-EMF and non-ELF-EMF groups were treated for the same amount of time (15 minutes). The non-ELF-EMF subjects were given only sham exposure. The pelvic girdle of the patients was exposed to the electromagnetic field, because exposure of the head to ELF-EMF can affect the activation of the epilepsy focus in the brain. The same therapeutic program was used for both subject groups. This consisted of aerobic exercise $(30 \mathrm{~min})$, neurophysiological routines $(60 \mathrm{~min})$, and psychological therapy (15 min). Poststroke patients with moderate stroke severity according to NIHSS scores of $4.9 \pm 3.1$ in the ELF-EMF group (aged $48.8 \pm 7.7$ ) and $5.4 \pm 2.9$ (aged $44.8 \pm 8.0$ ) in the nonELF-EMF group were enrolled in the study. Table 1 shows the clinical and demographic characteristics. Participants with haemorrhagic stroke, dementia, chronic or significant acute inflammatory factors, decreased consciousness, and/ 
TABLE 1: Clinical demographic characteristics.

\begin{tabular}{|c|c|c|c|c|}
\hline & & $\begin{array}{c}\text { Control } \\
n=23\end{array}$ & $\begin{array}{c}\text { Study group } \\
n=25\end{array}$ & $p$ \\
\hline \multirow{3}{*}{ Demographics } & Age $($ mean $\pm S D)$ & $44.8 \pm 7.7$ & $48.0 \pm 8.0$ & 0.84 \\
\hline & Sex: man versus female (\%) & 48 versus 52 & 60 versus 40 & 0.27 \\
\hline & Living alone (\%) & 32.1 & 34.2 & 0.59 \\
\hline \multirow{4}{*}{ Vascular risk } & Hypertension (\%) & 97.3 & 98.5 & 0.07 \\
\hline & Diabetes (\%) & 31.4 & 39.2 & 0.21 \\
\hline & Dyslipidemia (\%) & 78.8 & 72.2 & 0.7 \\
\hline & $\mathrm{BMI} \geq 30(\%)$ & 21 & 34 & 0.78 \\
\hline \multirow{3}{*}{ Concomitant medications } & Antidepressants (\%) & 29 & 34 & 0.5 \\
\hline & ASA (\%) & 70 & 65 & 0.42 \\
\hline & NSAID (\%) & 25 & 27 & 0.8 \\
\hline \multirow{3}{*}{ Stroke characteristics } & Weeks since stroke $($ mean $\pm \mathrm{SD})$ & $3.9 \pm 0.6$ & $3.2 \pm 0.4$ & \\
\hline & NIHSS scores $($ mean \pm SD) & $5.4 \pm 2.9$ & $4.9 \pm 3.1$ & \\
\hline & $\mathrm{ADL}($ mean $\pm \mathrm{SD})$ & $8.89 \pm 2.87$ & $9.95 \pm 2.35$ & 0.22 \\
\hline \multirow{3}{*}{ Lesion location } & Anterior $(n)$ & 3 & 5 & \\
\hline & Posterior $(n)$ & 7 & 6 & \\
\hline & Intermediate $(n)$ & 13 & 14 & \\
\hline \multirow{2}{*}{ Lesion side } & Left $(n)$ & 15 & 13 & \\
\hline & Right $(n)$ & 8 & 12 & \\
\hline
\end{tabular}

or neurological illness other than stroke in their medical prestroke history were excluded. The subjects had undergone neurorehabilitation for 4 weeks in Neurorehabilitation Ward III of the General Hospital in Lodz, Poland, as well as internal and neurological examinations. The Bioethics Committee of the Faculty of Biology and Environmental Protection of The University of Lodz, Poland, approved the protocol with resolution numbers 28/KBBN-UŁ/II/2015 and 13/KBBN$\mathrm{U} / \mathrm{II} / 2016$. All participants provided written informed consent prior to participation. Depression was screened in both groups using the Geriatric Depression Scale (GDS). Cognitive status was estimated in a Mini-Mental State Examination (MMSE), and functional status using the Barthel Index of Activities of Daily Living (ADL). The GDS, ADL, and MMSE were administered either on the same day as the blood sampling or on the afternoon before.

2.3. Magnetronic MF10 Devices. ELF-EMF therapy was performed by a Magnetronic MF10 generator as per accepted guidelines. This device is able to produce pulses in rectangular, trapezoid, and sinusoidal shapes. The pulses were applied using an AS-550 applicator (EiE, Otwock, Poland), which has the following properties: $550 \mathrm{~mm}$ in diameter, $270 \mathrm{~mm}$ in length, and 5 layers of 187 turns of $1.45 \mathrm{~mm}$ twin-parallel wires. Magnetic induction was set at $5 \mathrm{mT}$. The electromagnetic field intensity was not uniformed; its distribution is vertical, while the induction coils are set horizontally. Induction of the electromagnetic field of $5 \mathrm{mT}$ is present at the geometric center of the applicator, and the value increases in the proximity to the surface about $7 \mathrm{mT}$. Other factors that could affect EMF were eliminated (electronic measuring instruments occurring in rehabilitation room and other electronic equipment).
2.4. Immunodetection of 3-Nitrotyrosine by c-ELISA. Levels of 3-NT-containing proteins in plasma were determined using a modified c-ELISA method, as described by Khan et al. [14]. 96-well microtiter plates were coated with nitro-fibrinogen (nitro-Fg) $(1 \mathrm{mg} / \mathrm{mL})$ and kept overnight at $4^{\circ} \mathrm{C}$. Concentrations of nitrated proteins inhibiting the binding of antinitrotyrosine antibodies were assessed from the standard curve (10-100 nM nitro-Fg equivalents) and expressed as nitro-Fg equivalents [15].

2.5. Nitrate/Nitrite Estimation. Plasma samples were diluted twice before the measurement of nitrate/nitrite concentration using a Nitrate/Nitrite Colorimetric Assay Kit (Cayman Chemical Company, USA), based on the two-step Griess method. In the first step, the nitrate is converted to nitrite with nitrate reductase, while in the second step, after addition of the Griess reagent, the nitrite is converted to a deep purple azo compound. The absorbance measurement was performed at $540 \mathrm{~nm}$ in a 96-well microplate reader (SPECTROstarNano, BMG Labtech, Ortenberg, Germany) [16].

2.6. Determination of NOS2 Expression in Whole Blood Samples. RNA was isolated from the frozen whole blood samples $\left(-80^{\circ} \mathrm{C}\right)$, in accordance with the manufacturer's protocol using TRI Reagent ${ }^{\circledR}$ (Sigma-Aldrich, USA). The aqueous phase was purified in accordance with the manufacturer's protocol using an InviTrap Spin Universal RNA Mini Kit (Stratec Biomedical Systems, Germany). The purity and quantity of isolated RNA were assessed using a Synergy HTX Multi-Mode Microplate Reader equipped with a Take3 Micro-Volume Plate and connected to a PC running Gen5 Software (BioTek Instruments Inc., Winooski, VT, USA). Isolated RNA $(20 \mathrm{ng} / \mu \mathrm{L})$ was transcribed onto cDNA with 


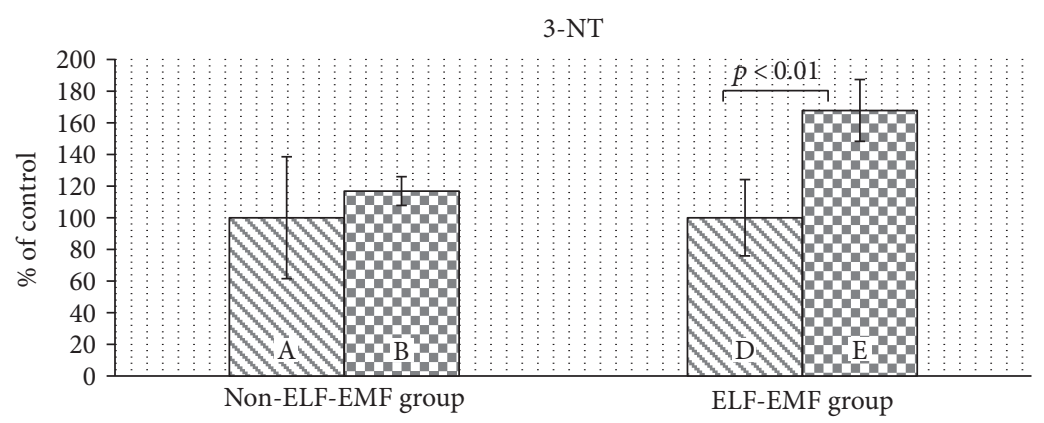

Before treatment

回 After 10 treatments

Figure 2: The comparison of 3-NT levels in plasma proteins obtained from the ELF-EMF group versus those from the non-ELF-EMF group. Statistical significance between the ELF-EMF and non-ELF-EMF groups: B versus D $(p<0.05)$.

a High-Capacity cDNA Reverse Transcription Kit (Applied Biosystems ${ }^{\mathrm{TM}}$, Waltham, MA, USA). Quantitative assays were executed using a TaqMan Hs01075529_m1 probe for human NOS2 genes and an Hs02786624_g1 for endogenous control, which was GAPDH (Life Technologies). Reactions were carried out using a TaqMan Universal Master Mix II, without UNG (Life Technologies) in a BioRad CFX96 realtime PCR system (BioRad Laboratories, Hercules, CA, USA), all in accordance with the manufacturers' protocols. Relative expression of NOS2 was obtained using the equation $2^{-\Delta \mathrm{Ct}}$, where $\Delta \mathrm{Ct}$ is the threshold cycle $(\mathrm{Ct})$ value for the target gene minus $\mathrm{Ct}$ values obtained for the housekeeping gene GAPDH [17].

2.7. Determination of TNF $\alpha$. Measurements of human tumour necrosis factor alpha (TNF $\alpha)$ in plasma samples were made with a Human TNF $\alpha$ ELISA development kit (MABTECH, Cincinnati, OH, USA), in accordance with the manufacturer's protocol. The combination of two coating antibodies (TNF3 and TNF4) were used for the analysis. The absorbance was measured at $450 \mathrm{~nm}$, and TNF $\alpha$ concentration was expressed as $\mathrm{pg} / \mathrm{mL}$ [18].

2.8. Data Analysis. Biochemical and clinical data were expressed as mean \pm SD. All measurements were executed in duplicate. Output value (100\%) was determined for each measured parameter of each patient before treatment. Data from tests performed on the same study subjects after therapy constituted a percentage of the output value. Percentage values were presented as mean \pm SD. Statistical analyses were performed using the Statistica 12 statistical software (StaftSoft Inc.). A Shapiro-Wilk test was used to analyse for normality. A paired Student $t$-test was used to the calculate differences between the values obtained for subjects before and after therapy, whereas unpaired Student $t$-test or Mann-Whitney $U$ tests were used to determine differences between the ELFEMF and non-ELF-EMF groups. $p$ values of 0.05 were accepted as statistically significant for all analyses.

\section{Results}

Our comparative analysis demonstrated an increased level of 3-nitrotyrosine (3-NT) $(p<0.05)$ (Figure 2$)$ and an elevated nitrate/nitrite concentration $(p<0.01)$ (Figure 3$)$ in the plasma of patients from the ELF-EMF group. The gain in the 3-NT level was significantly higher with an increased amount of sessions (Figure 2). In the non-ELF-EMF group, we saw that the effect of rehabilitation on nitrative stress was largely weaker and not statistically significant $(p>0.05)$ (Figures 2 and 3 ). The 3-NT level increased more in the ELF-EMF group than in the non-ELF-EMF after 10 treatments $(68 \%$ versus $17 \%$, $p<0.05$ ) (Figure 2). The level of nitrate/nitrite in the nonELF-EMF group even decreased after 10 treatments (although not statistically significantly) (Figure 3 ).

In the next set of experiments, we determined the effect of magnetotherapy on gene expression in the whole blood samples of NOS2 mRNA. Its expression was unmeasurable in $35 \%$ of subjects from both the ELF-EMF and non-ELF-EMF groups. We observed a statistically insignificant decrease in the level of NOS2 mRNA expression after treatment in both the ELF-EMF and non-ELF-EMF groups (Figure 4).

Subsequently, we determined the concentration of proinflammatory cytokine TNF $\alpha$. We found that the concentration of TNF $\alpha$ was comparable before treatment in both the ELFEMF and non-ELF-EMF-groups. The cytokine level did not change in either groups after rehabilitation (Figure 5).

The ADL, MMSE, and GDS were used to evaluate the functional and mental status of poststroke patients undergoing rehabilitation. We demonstrated that treatment using ELF-EMF improves their clinical parameters, particularly in cognitive and psychosomatic functions.

Motor abilities estimated by ADL score changed at similar levels in both groups, with the observed improvement being statistically significant in all rehabilitated patients $(p<0.001)$ (Table 2).

The baseline MMSE values before treatment in both groups were comparable, but statistically different $(p<0.05)$ after rehabilitation. After 2 weeks of rehabilitation, MMSE parameters improved markedly in the ELF-EMF group $(p=0.002)$, while a small increase in the non-ELF-EMF group was not statistically significant $(p=0.2)$ (Table 2).

Depression syndrome expressed by GDS improved significantly in both groups after rehabilitation. However, the $\triangle$ GDS value reached about a $60 \%$ lower result in the ELF-EMF group than in the non-ELF-EMF group 


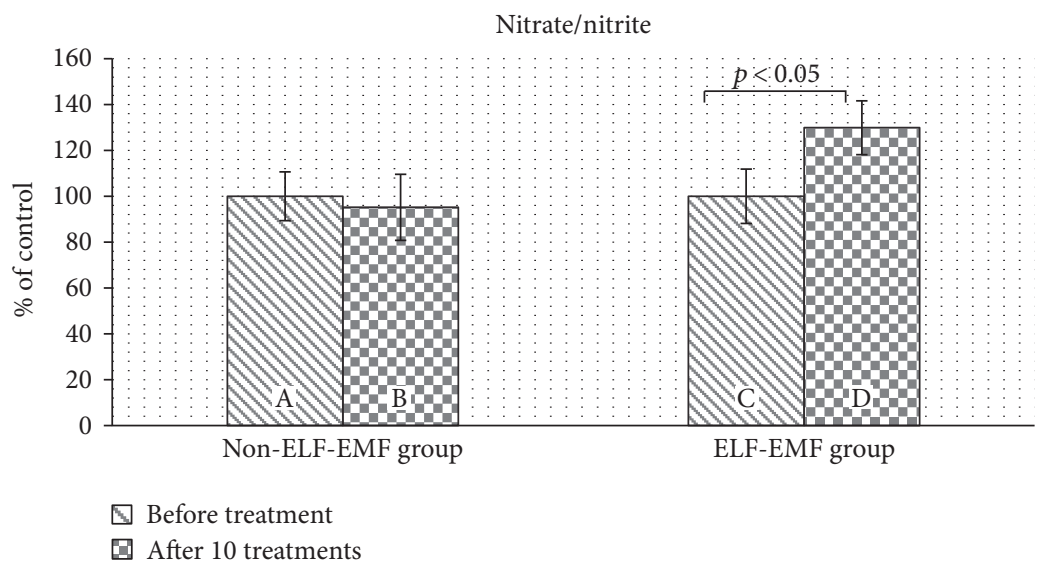

FIGURE 3: The comparison of nitrate/nitrite levels in plasma proteins obtained from the ELF-EMF group versus those from the non-ELF-EMF group. Statistical significance between ELF-EMF and non-ELF-EMF groups: B versus D $(p<0.05)$.

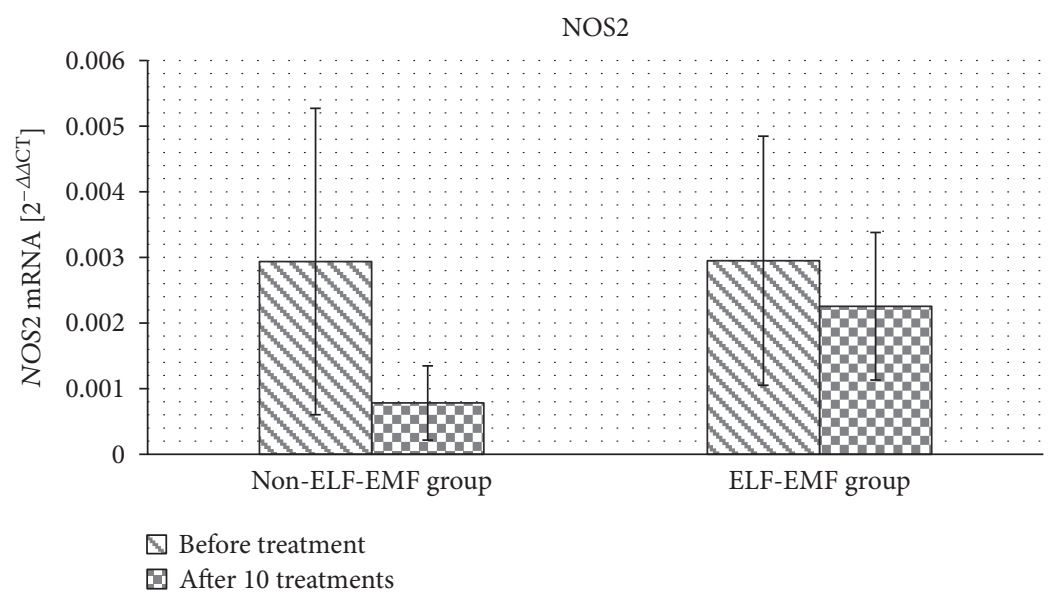

FIGURE 4: The comparison of NOS2 mRNA expression obtained from the ELF-EMF group versus that from the non-ELF-EMF group.

$(p=0.018)$, starting from a similar base level in both groups $(p>0.05)$ (Table 2).

\section{Discussion}

In this study, we provide the evidence that application of extremely low-frequency electromagnetic field increases nitric oxide generation and its metabolism, as well as improving the effectiveness of poststroke ischemic patients' treatments.

Ischemic stroke is one of the major causes of morbidity and mortality in the world's population and is one of the main causes of long-term disability. The mechanisms of neurological function recovery after brain injury associated with neuroplasticity (cortical reorganization) are still insufficiently understood. Poststroke neurorehabilitation is designed to provide external stimuli, improving the effectiveness of compensatory plasticity [19].

In the central nervous system, $\mathrm{NO}$ is both a pre- and postsynaptic signal molecule. The activity of NO is associated with a cGMP-mediated signalling cascade. The presynaptic excitatory action of $\mathrm{NO}$ is related to the phosphorylation of synaptophysin by the cGMP-dependent protein kinase $\mathrm{G}$ (PKG) pathway and the subsequent potentates of glutamatergic neurotransmission [20]. On the other hand, NO causes a neurotransmission inhibition through gammaaminobutyric acid- (GABA-) ergic synaptic communication. It is associated with ion exchange and regulation of membrane excitation [21, 22]. Moreover, NO as an important vasodilation factor mediates neurovascular coupling. The enlargement of vessel diameter is caused by increasing metabolic consumption as a result of neuronal activity. Neurovascular coupling maintains functional and structural brain integrity [23].

This study was designed to investigate the impact of ELFEMF on the metabolism of nitric oxide in the rehabilitation of acute poststroke patients.

In our study, we demonstrate that poststroke rehabilitation increases the level of 3-NT and nitrate/nitrite concentrations. Due to its vasodilating and proangiogenic effects, NO serves as a protective function during cerebral ischemia. $\mathrm{Su}$ et al. investigated the role of simvastatin-regulated TRPV1 receptors (transient receptor potential vanilloid type 1) in NO bioavailability, activation of eNOS, and angiogenesis in 


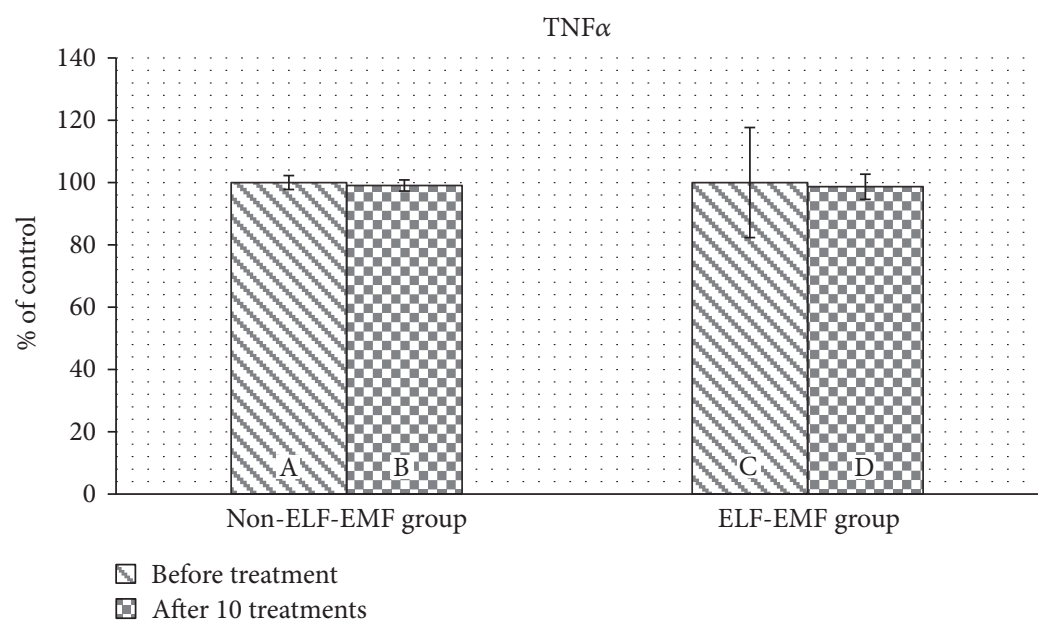

FIGURE 5: The comparison of TNF $\alpha$ levels in plasma proteins obtained from the ELF-EMF group versus those from the non-ELF-EMF group.

TABLE 2: Clinical parameters: ADL, MMSE, and GDS measured in the ELF-EMF and non-ELF-EMF groups. Data presented as the delta of a clinimetric scale before and after the standard series of treatments $(\triangle \mathrm{ADL}=$ the increase of $\mathrm{ADL} ; \triangle \mathrm{MMSE}=$ the increase of MMSE; and $\triangle \mathrm{GDS}=$ the decrease of GDS).

\begin{tabular}{ccccc}
\hline & & $\begin{array}{c}\text { Non-ELF-EMF } \\
\text { group }\end{array}$ & $\begin{array}{c}\text { ELF-EMF } \\
\text { group }\end{array}$ & $p$ \\
\hline \multirow{4}{*}{ ADL } & Before & 8.99 & 9.95 & 0.378 \\
& treatment & & & \\
& After treatment & 14.3 & 16.12 & 0.429 \\
& $\Delta$ & 5.31 & 6.17 & 0.194 \\
\multirow{4}{*}{ MMSE } & After treatment & 23.94 & 26.28 & 0.047 \\
& $\Delta$ & 1.19 & 3.28 & 0.036 \\
& Before & 22.75 & 23.00 & 0.873 \\
& $p$ & 0.204 & 0.002 & \\
\hline \multirow{4}{*}{ GDS } & Before & 11.38 & 13.00 & 0.054 \\
& After treatment & 9.13 & 6.72 & 0.049 \\
& $\Delta$ & 2.25 & 6.28 & 0.018 \\
& treatment & 0.009 & 0.001 & \\
\hline
\end{tabular}

mice. They demonstrated that simvastatin causes an influx of calcium ions through the TRPV1-TRPA1 (transient receptor potential ankyrin 1) pathway, which then causes activation of CaMKII $\left(\mathrm{Ca}^{2+} /\right.$ calmodulin-dependent protein kinase II). This then enhances the formation of the TRPV1-eNOS complex, which also includes CaMKII, AMPK (5'AMP-activated protein kinase), and Akt (protein kinase B), which leads to activation of eNOS, production of NO, and thus the promotion of endothelial angiogenesis [24]. There have been numerous reports of the protective effects of $\mathrm{NO}$ against inflammation and oxidative stress [25]. Transgenic eNOSdeficient mice demonstrated a more extensive infarct of the middle cerebral artery (MCA), compared to controls [26].
NO effects on the regulation of endothelial integrity, anti-inflammatory and anti-apoptotic effects, as well as maintenance of cerebral blood flow, inhibition of platelet aggregation, and reduction of leukocyte adhesion [25, 27]. Khan et al. studied structurally different NO donors as agents of cerebrovascular protection in experimentally induced stroke in rats. They showed that NO donors promote cerebral blood flow through S-nitrosylation and may be an effective drug for acute stroke $[28,29]$.

Furthermore, Greco et al. proved the protective effect of nitroglycerin (donors of $\mathrm{NO}$ ) on cerebral damage induced by MCA occlusion in Wistar rats. They observed a significant reduction in stroke volume in preinjected rats compared to their control group, which confirms the protective effect of nitroglycerin in vivo. They speculated that the mechanism of action is associated with the generation of a complex chain of phenomena, triggering activation of apoptosis and subsequent activation of antiapoptotic responses [30].

The biological action of ELF-EMF is still being investigated. It is suggested that ELF-EMF has an impact on the physicochemical properties of water, the liquid crystal structure generated by cholesterol, and its derivatives [31,32]. Changes in ion balance caused by ELF-EMF appeal to the structure of tissue with piezoelectric and magnetostrictive properties, free radicals, diamagnetic molecules, and uncompensated magnetic spins of paramagnetic elements [33]. Therefore, ELFEMF causes depolarization of cells having the ability to spontaneously depolarize, predominantly through $\mathrm{Ca}^{2+}$ influx [34]. In our previous study, we investigated the effect of ELFEMF on oxidative stress in patients after ischemic stroke. We demonstrated that ELF-EMF causes activation of antioxidant enzymes [35], which leads to reduction of the oxidative modification of plasma protein (this is detailed in an article published in Advances in Clinical and Experimental Medicine). As a highly reactive molecule, $\mathrm{NO}$ can also regulate the level of oxidative stress. Through the covalent interaction, NO influences the activity of various enzymes. Mechanisms of this modulation can be varied: NO reacts with coenzymes and active centers containing metal ions and interacts with cysteine residues of proteins [36]. 
In the current study, we observed that in the ELF-EMF group, the level of plasma 3-NT was increased (Figure 2). The formation of 3-NT in protein molecules occurs in vivo by the action of nitrating agents on the polypeptide chain. The formation of 3-NT is mainly attributed to $\mathrm{NO}$ and superoxide anions $\left(\mathrm{O}_{2}^{-\cdot}\right)$, which react rapidly to form peroxynitrite $\left(\mathrm{ONOO}^{-}\right)$. This is one of the major oxidizing and nitrating agents produced in vivo in acute and chronic inflammation, as well as in ischemia/reperfusion. Endothelial cells, macrophages, and neutrophils release large amounts of $\mathrm{NO}$ and $\mathrm{O}_{2}^{-}$. Thus, increased amounts of NO contribute to the creation of 3-NT [37].

To investigate the effect of ELF-EMF on NO metabolism, we determined nitrate/nitrite concentrations in plasma. We showed that in the ELF-EMF group, the level of nitrate/ nitrite compounds in plasma increased after treatment (Figure 3), and these results correspond with the data presented by Chung et al. [38]. The authors investigated the effects of ELF-EMF $(60 \mathrm{~Hz}, 2 \mathrm{mT})$ on the level of $\mathrm{NO}$, biogenic amines, and amino acid neurotransmitters in the hippocampus, cortex, thalamus, cerebellum, and striatum in rats. They found a significant increase in NO concentration in the hippocampus, thalamus, and striatum. Moreover, ELF-EMF also caused a change in the level of biogenic amines and amino acid neurotransmitters in the brain. However, the observed effect and range were different, depending on the brain area. Balind et al. determined the effect of ELFEMF $(50 \mathrm{~Hz}, 0.5 \mathrm{mT})$ on oxidative stress in gerbils with induced cerebral ischemia. They measured the level of NO using the Griess reagent and showed an increased level of NO, provoked by electromagnetic fields. Moreover, ELFEMF reduces oxidative stress generated during cerebral ischemia, thus leading to a decrease in the damaged brain tissue [39].

$\mathrm{NO}$ is produced from $\mathrm{L}$-arginine with the involvement of nitric oxide synthase. Three NOS isoforms are expressed in different tissues. Although, in the blood, only NOS2 is expressed, in 35\% of the subjects in both the ELF-EMF and non-ELF-EMF groups, mRNA expression of NOS2 was under detection. In the remaining patients, the expression of NOS 2 had not significantly changed after treatment. The NOS2 gene in fact encodes for iNOS, which is primarily activated during inflammation. In order to exclude deeper inflammation, we measured the concentration of $\mathrm{TNF} \alpha$, one of the main proinflammatory cytokines. TNF $\alpha$ is a pleiotropic cytokine that is involved in nearly all phenomena of inflammatory responses: initiating chemokine synthesis, promoting the expression of adhesion molecules, promoting the maturation of dendritic cells, and inducing the production of inflammatory mediators and other proinflammatory cytokines [40]. TNF $\alpha$ stimulates collagenase synthesis in synovial fibroblasts and synovial cartilage chondrocytes and activates osteoclasts, leading to joint cartilage damage, hypertrophy, bone resorption and erosion, and angiogenesis. It also activates monocytes and macrophages, enhancing their cytotoxicity and stimulating cytokine production. Chemokines and growth factors are responsible for $\mathrm{T}$ cell proliferation, proliferation and differentiation of B lymphocytes, and the release of inflammatory cytokines by the lymphocytes. Moreover, in the hypothalamus, $\mathrm{TNF} \alpha$ stimulates prostaglandin $\mathrm{E}$ and IL-1 synthesis [41]. Pena-Philippides et al. investigated the effect of pulsed electromagnetic fields on injury size and neuroinflammation in mice after middle cerebral artery occlusion (MCAO). They found, using magnetic resonance imaging (MRI), that EMF reduced infarct size, as well as changed expression of genes encoding pro- and antiinflammatory cytokines in the hemisphere with ischemic injury. After EMF exposure, genes encoding IL- $1 \alpha$ and TNF superfamily were downregulated, while $I L-10$ expression was upregulated. Thus, the authors suggested that application of EMF to poststroke patients could have been beneficial through anti-inflammatory effect and reduction of injury size [42].

On the basis of our results, we suggest that the observed increase in NO level is associated with nNOS and/or eNOS activities, but not with iNOS expression. Our research is consistent with evidence shown by Cho et al., who established that ELF-EMF $(60 \mathrm{~Hz}, 2 \mathrm{mT})$ increased the expression and activation of nNOS in rat brains [43].

The activities of nNOS and eNOS depend on calcium ions. There are many reports that the biological effect of ELF-EMF is related to the control of calcium channels [44-48]. In view of these findings, the observed mechanism of increased NO generation and metabolism may be associated with calcium-ion flux.

Additionally, we noticed that ELF-EMF treatment enhances the effectiveness of poststroke rehabilitation (Table 2). Some researchers suggest that electromagnetic fields have a beneficial effect on ischemic/reperfusion injury, and in some places, therapeutic programs using ELF-EMF are considered to be standard therapy for poststroke patients $[49,50]$. The beneficial effects of ELFEMF include the following: improvement in the transport of cellular and mitochondrial membranes; normalization of blood rheological values; counteraction of tissue oxidation; intensification of regenerative processes; stimulation of axon growth in undamaged neurons; intensification of neuronal dissociation and differentiation; reduction of stress-induced emotional reactions and free radicals; acceleration of the return of fibre function in functional disorders; reduction of periapical scarring; and increase of the level of energetic substances in the brain tissue and erythrocytes [48-53]. Grant et al. estimated the impact of low-frequency pulsed electromagnetic field on cerebral ischemia in rabbit. They observed using MRI that exposure to electromagnetic field caused extenuation of cortical ischemia oedema and reduction of neuronal injury in cortical area [54].

In conclusion, ELF-EMF therapy increases the metabolism and generation of $\mathrm{NO}$, which has both neuroprotective and cytotoxic properties. An increase in NO level is probably associated with nNOS and/or eNOS activities, but not with iNOS expression, which increases mainly during inflammation. We suggested that in poststroke patients, NO demonstrated a protective effect due to significant improvement in patient functional status. Thus, our studies promote the validity of this method in poststroke rehabilitation therapy. 


\section{Conflicts of Interest}

The authors declare that there is no conflict of interest regarding the publication of this article.

\section{Acknowledgments}

This study was supported by the Department of General Biochemistry, Faculty of Biology and Environmental Protection, University of Lodz (no. 506/1136), and Laboratory of Medical Genetics, Faculty of Biology and Environmental Protection, University of Lodz (no. B161100000004601), and Grants for Young Scientists and PhD Students, Faculty of Biology and Environmental Protection, University of Lodz (B1611000001145.02).

\section{References}

[1] N. Townsend, L. Wilson, P. Bhatnagar, K. Wickramasinghe, M. Rayner, and M. Nichols, "Cardiovascular disease in Europe: epidemiological update 2016," European Heart Journal, vol. 37, no. 42, pp. 3232-3245, 2016.

[2] X. Li, L. Su, X. Zhang et al., "Ulinastatin downregulates TLR4 and NF-kB expression and protects mouse brains against ischemia/reperfusion injury," Neurological Research, vol. 13, pp. 1-7, 2017.

[3] T. Klarner, T. S. Barss, Y. Sun, C. Kaupp, P. M. Loadman, and E. P. Zehr, "Long-term plasticity in reflex excitability induced by five weeks of arm and leg cycling training after stroke," Brain Sciences, vol. 6, no. 4, 2016.

[4] Y. Cheng, Y. Dai, X. Zhu et al., "Extremely low-frequency electromagnetic fields enhance the proliferation and differentiation of neural progenitor cells cultured from ischemic brains," Neuroreport, vol. 26, no. 15, pp. 896-902, 2015.

[5] N. Cichon, A. K. Olejnik, E. Miller, and J. Saluk, "The multipotent action of magnetic fields," Biologia, vol. 71 , no. 10, pp. 1103-1110, 2016.

[6] J. A. Robertson, A. W. Thomas, Y. Bureau, and F. S. Prato, "The influence of extremely low frequency magnetic fields on cytoprotection and repair," Bioelectromagnetics, vol. 28, no. 1, pp. 16-30, 2007.

[7] S. Kumar, R. K. Singh, and T. R. Bhardwaj, "Therapeutic role of nitric oxide as emerging molecule," Biomedicine \& Pharmacotherapy, vol. 85, pp. 182-201, 2017.

[8] W. K. Alderton, C. E. Cooper, and R. G. Knowles, "Nitric oxide synthases: structure, function and inhibition," The Biochemical Journal, vol. 357, Part 3, pp. 593-615, 2001.

[9] H. Li and T. L. Poulos, "Structure-function studies on nitric oxide synthases," Journal of Inorganic Biochemistry, vol. 99, pp. 293-305, 2005.

[10] J. Lei, Y. Vodovotz, E. Tzeng, and T. R. Billiar, "Nitric oxide, a protective molecule in the cardiovascular system," Nitric Oxide, vol. 35, pp. 175-185, 2013.

[11] M. Rasool, M. A. Ashraf, A. Malik et al., "Comparative study of extrapolative factors linked with oxidative injury and antiinflammatory status in chronic kidney disease patients experiencing cardiovascular distress," PLoS One, vol. 12, no. 2, article e0171561, 2017.

[12] A. V. Kozlov, S. Bahrami, H. Redl, and C. Szabo, "Alterations in nitric oxide homeostasis during traumatic brain injury,"
Biochimica et Biophysica Acta (BBA) - Molecular Basis of Disease, 2017, In press.

[13] T. Mika, Metodyka Magnetoterapii, T. Mika and W. Kasprzak, Eds., pp. 337-339, Fizykoterapia, Wydawnictwo Lekarskie PZWL. Warszawa, 2013.

[14] J. Khan, D. M. Brennan, N. Bradley, B. Gao, R. Bruckdorfer, and M. Jacobs, Part 2, "3-Nitrotyrosine in the proteins of human plasma determined by an ELISA method," The Biochemical Journal, vol. 330, pp. 795-801, 1998.

[15] J. Kolodziejczyk, J. Saluk-Juszczak, and B. Wachowicz, "L-Carnitine protects plasma components against oxidative alterations," Nutrition, vol. 27, no. 6, pp. 693-699, 2011.

[16] A. É. Declèves, I. Jadot, V. Colombaro et al., "Protective effect of nitric oxide in aristolochic acid-induced toxic acute kidney injury: an old friend with new assets," Experimental Physiology, vol. 101, no. 1, pp. 193-206, 2016.

[17] K. Bobińska, J. Szemraj, P. Czarny, and P. Gałecki, "Expression and activity of metalloproteinases in depression," Medical Science Monitor, vol. 22, pp. 1334-1341, 2016.

[18] O. Désy, D. Carignan, M. Caruso, and P. O. de Campos-Lima, "Methanol induces a discrete transcriptional dysregulation that leads to cytokine overproduction in activated lymphocytes," Toxicological Sciences, vol. 117, no. 2, pp. 303-313, 2010.

[19] C. Allman, U. Amadi, A. M. Winkler et al., "Ipsilesional anodal tDCS enhances the functional benefits of rehabilitation in patients after stroke," Science Translational Medicine, vol. 8, no. 330, p. 330re1, 2016.

[20] H. G. Wang, F. M. Lu, I. Jin et al., "Presynaptic and postsynaptic roles of $\mathrm{NO}, \mathrm{cGK}$, and RhoA in long-lasting potentiation and aggregation of synaptic proteins," Neuron, vol. 45, pp. 389-403, 2005.

[21] Y. R. Yang, J. H. Jung, S. J. Kim et al., "Forebrain-specific ablation of phospholipase $\mathrm{C} \gamma 1$ causes manic-like behavior," Molecular Psychiatry, 2017.

[22] C. D. Fekete, R. U. Goz, S. Dinallo et al., "In vivo transgenic expression of collybistin in neurons of the rat cerebral cortex," The Journal of Comparative Neurology, vol. 525, no. 5, pp. 1291-1311, 2017.

[23] Z. Ungvari, S. Tarantini, P. Hertelendy et al., "Cerebromicrovascular dysfunction predicts cognitive decline and gait abnormalities in a mouse model of whole brain irradiation-induced accelerated brain senescence," Geroscience, vol. 39, no. 1, pp. 33-42, 2017.

[24] K. H. Su, S. J. Lin, J. Wei et al., "The essential role of transient receptor potential vanilloid 1 in simvastatin-induced activation of endothelial nitric oxide synthase and angiogenesis," Acta Physiologica (Oxford, England), vol. 212, no. 3, pp. 191204, 2014.

[25] G. Cirino, S. Fiorucci, and W. C. Sessa, "Endothelial nitric oxide synthase: the Cinderella of inflammation?," Trends in Pharmacological Sciences, vol. 24, pp. 91-95, 2003.

[26] Z. Huang, P. L. Huang, J. Ma et al., "Enlarged infarcts in endothelial nitric oxide synthase knockout mice are attenuated by nitro-L-arginine," Journal of Cerebral Blood Flow and Metabolism, vol. 16, pp. 981-987, 1996.

[27] H. Li and U. Forstermann, "Nitric oxide in the pathogenesis of vascular disease," The Journal of Pathology, vol. 190, pp. 244-254, 2000.

[28] M. Khan, M. Jatana, C. Elango, A. S. Paintlia, A. K. Singh, and I. Singh, "Cerebrovascular protection by various nitric oxide 
donors in rats after experimental stroke," Nitric Oxide, vol. 15, no. 2, pp. 114-124, 2006.

[29] M. Khan, B. Sekhon, S. Giri et al., "S-Nitrosoglutathione reduces inflammation and protects brain against focal cerebral ischemia in a rat model of experimental stroke," Journal of Cerebral Blood Flow and Metabolism, vol. 25, pp. 177-192, 2005.

[30] R. Greco, D. Amantea, F. Blandini et al., "Neuroprotective effect of nitroglycerin in a rodent model of ischemic stroke: evaluation of Bcl-2 expression," International Review of Neurobiology, vol. 82, pp. 423-435, 2007.

[31] M. Sulpizio, S. Falone, F. Amicarelli et al., "Molecular basis underlying the biological effects elicited by extremely lowfrequency magnetic field (ELF-MF) on neuroblastoma cells," Journal of Cellular Biochemistry, vol. 112, pp. 3797-3806, 2011.

[32] G. Yi, J. Wang, X. Wei et al., "Effects of extremely lowfrequency magnetic fields on the response of a conductancebased neuron model," International Journal of Neural Systems, vol. 24, no. 1, article 1450007, 2014.

[33] F. Brisdelli, F. Bennato, A. Bozzi, B. Cinque, F. Mancini, and R. Iorio, "ELF-MF attenuates quercetin-induced apoptosis in K562 cells through modulating the expression of Bcl-2 family proteins," Molecular and Cellular Biochemistry, vol. 397, no. 1-2, pp. 33-43, 2014.

[34] C. Morgado-Valle, L. Verdugo-Díaz, D. E. García, C. MoralesOrozco, and R. Drucker-Colín, "The role of voltage-gated $\mathrm{Ca}^{2+}$ channels in neurite growth of cultured chromaffin cells induced by extremely low frequency (ELF) magnetic field stimulation," Cell and Tissue Research, vol. 291, no. 2, pp. 217-230, 1998.

[35] N. Cichon, M. Bijak, E. Miller, and J. Saluk, "Extremely lowfrequency electromagnetic field (ELF-EMF) reduces oxidative stress and improves functional and psychological status in ischemic stroke patients," Bioeletromagtetics, vol. 38, no. 5, pp. 386-396, 2017.

[36] D. A. Wink, K. M. Miranda, M. G. Espey et al., "Mechanisms of the antioxidant effects of nitric oxide," Antioxidants \& Redox Signaling, vol. 3, no. 2, pp. 203-213, 2001.

[37] R. S. Ronson, M. Nakamura, and J. Vinten-Johansen, "The cardiovascular effects and implications of peroxynitrite," Cardiovascular Research, vol. 44, pp. 47-59, 1999.

[38] Y. H. Chung, Y. J. Lee, H. S. Lee et al., "Extremely low frequency magnetic field modulates the level of neurotransmitters," The Korean Journal of Physiology and Pharmacology, vol. 19, no. 1, pp. 15-20, 2015.

[39] B. S. Rauš, V. Selaković, L. Radenović, Z. Prolić, and B. Janać, "Extremely low frequency magnetic field $(50 \mathrm{~Hz}, 0.5 \mathrm{mT})$ reduces oxidative stress in the brain of gerbils submitted to global cerebral ischemia," PLoS One, vol. 9, no. 2, article e88921, 2014.

[40] P. Wu, F. Jia, B. Zhang, and P. Zhang, "Risk of cardiovascular disease in inflammatory bowel disease," Experimental and Therapeutic Medicine, vol. 13, no. 2, pp. 395-400, 2017.

[41] J. Godos, A. Biondi, F. Galvano et al., "Markers of systemic inflammation and colorectal adenoma risk: meta-analysis of observational studies," World Journal of Gastroenterology, vol. 23, no. 10, pp. 1909-1919, 2017.

[42] J. C. Pena-Philippides, Y. Yang, O. Bragina, S. Hagberg, E. Nemoto, and T. Roitbak, "Effect of pulsed electromagnetic field (PEMF) on infarct size and inflammation after cerebral ischemia in mice," Translational Stroke Research, vol. 5, no. 4, pp. 491-500, 2014.

[43] S. I. Cho, Y. S. Nam, L. Y. Chu et al., "Extremely low-frequency magnetic fields modulate nitric oxide signaling in rat brain," Bioelectromagnetics, vol. 33, no. 7, pp. 568-574, 2012.

[44] J. Walleczek, "Electromagnetic field effects on cells of the immune system: the role of calcium signaling," The FASEB Journal, vol. 6, pp. 3177-3185, 1992.

[45] C. Grassi, M. D'Ascenzo, A. Torsello et al., "Effects of $50 \mathrm{~Hz}$ electromagnetic fields on voltage-gated $\mathrm{Ca}^{2+}$ channels and their role in modulation of neuroendocrine cell proliferation and death," Cell Calcium, vol. 35, pp. 307-315, 2004.

[46] R. Piacentini, C. Ripoli, D. Mezzogori, G. B. Azzena, and C. Grassi, "Extremely low-frequency electromagnetic fields promote in vitro neurogenesis via upregulation of $\mathrm{Ca}_{\mathrm{v}} 1$ channel activity," Journal of Cellular Physiology, vol. 215, pp. 129-139, 2008.

[47] F. Gobba, D. Malagoli, and E. Ottaviani, "Effects of $50 \mathrm{~Hz}$ magnetic fields on fMLP-induced shape changes in invertebrate immunocytes: the role of calcium ion channels," Bioelectromagnetics, vol. 24, pp. 277-282, 2003.

[48] G. L. Craviso, S. Choe, P. Chatterjee, I. Chatterjee, and P. T. Vernier, "Nanosecond electric pulses: a novel stimulus for triggering $\mathrm{Ca}^{2+}$ influx into chromaffin cells via voltage-gated $\mathrm{Ca}^{2+}$ channels," Cellular and Molecular Neurobiology, vol. 30, pp. 1259-1265, 2010.

[49] A. Sieroñ and G. Cieslar, "Use of magnetic fields in medicine 15 years of personal experience," Wiadomości Lekarskie, vol. 56, pp. 434-441, 2003.

[50] M. Woldańska-Okońska and J. Czernicki, "Effect of low frequency magnetic fields used in magnetotherapy and magnetostimulation on the rehabilitation results of patients after ischemic stroke," Przeglad Lekarski, vol. 64, no. 2, pp. 74-77, 2007.

[51] F. Capone, M. Dileone, P. Profice et al., "Does exposure to extremely low frequency magnetic fields produce functional changes in human brain?," Journal of Neural Transmission (Vienna), vol. 116, no. 3, pp. 257-265, 2009.

[52] A. Miecznik, J. Czernicki, and J. Krukowska, "Influence of magnetic field of different characteristics on blood pressure in patients with back pain syndromes and hypertensive disease," Acta Bio-Optica et Informatica Medica, vol. 7, no. 1-2, pp. 9-13, 2001.

[53] V. Di Lazzaro, F. Capone, F. Apollonio et al., “A consensus panel review of central nervous system effects of the exposure to low-intensity extremely low-frequency magnetic fields," Brain Stimulation, vol. 6, no. 4, pp. 469-476, 2013.

[54] G. Grant, R. Cadossi, and G. Steinberg, "Protection against focal cerebral ischemia following exposure to a pulsed electromagnetic field," Bioelectromagnetics, vol. 15, no. 3, pp. 205-216, 1994. 


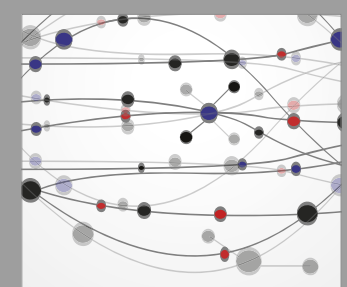

The Scientific World Journal
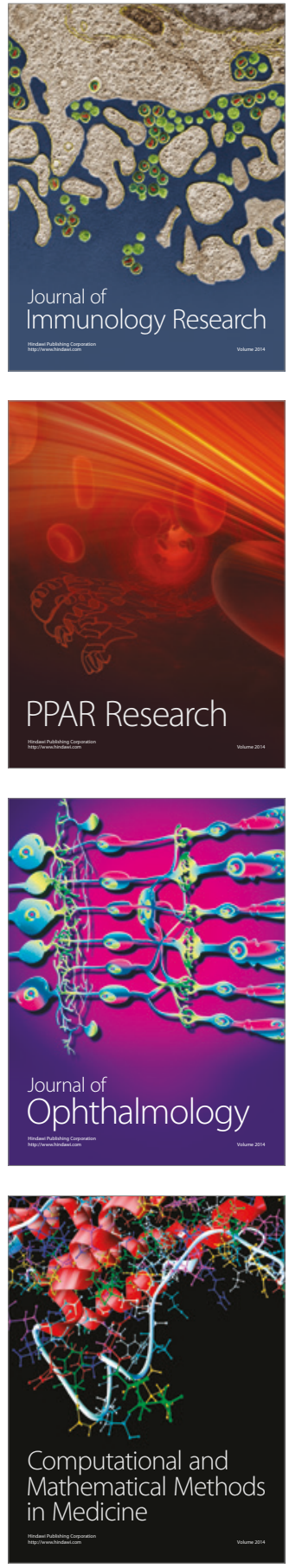

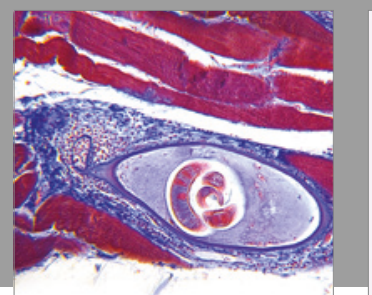

Gastroenterology Research and Practice
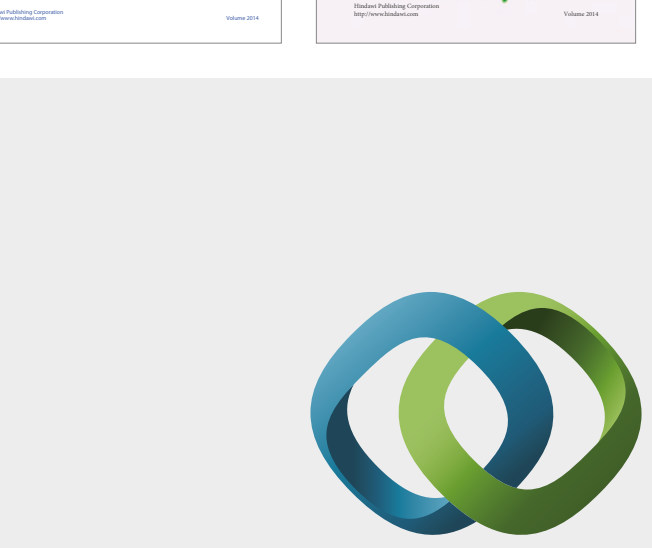

\section{Hindawi}

Submit your manuscripts at

https://www.hindawi.com
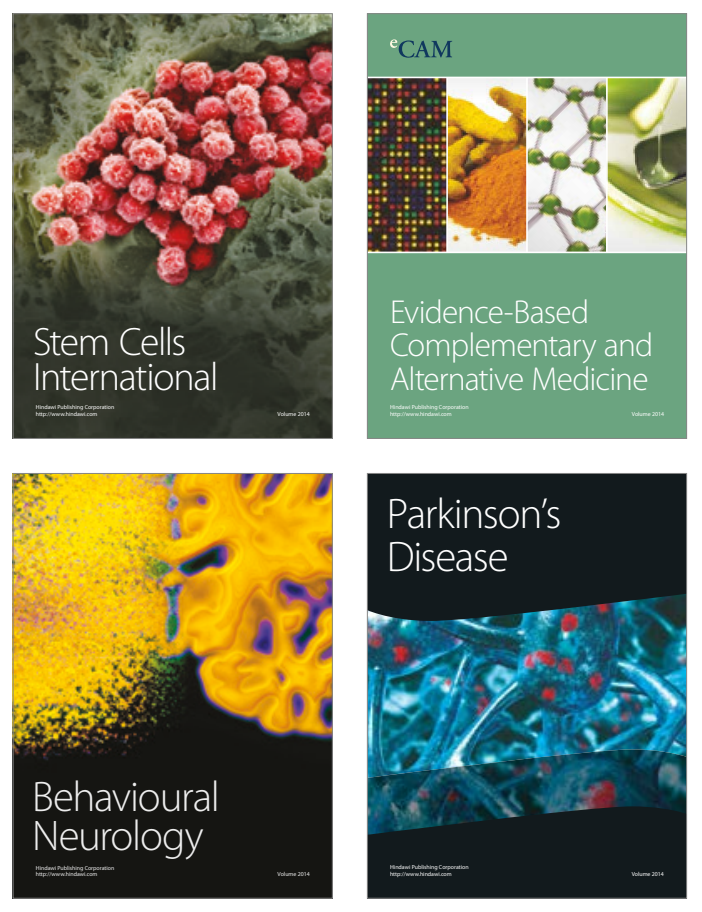
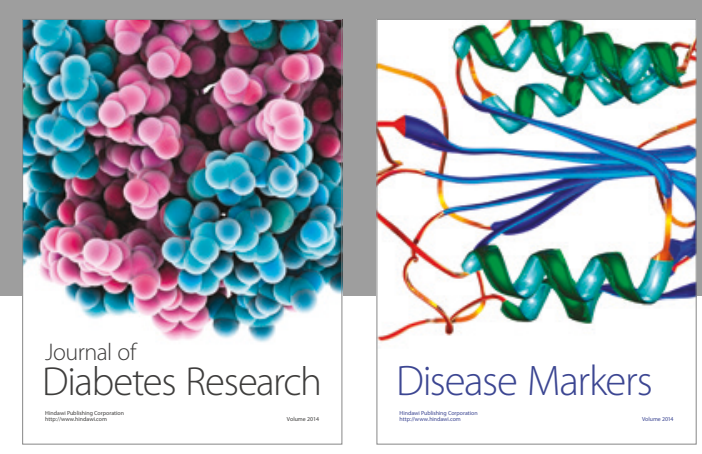

Disease Markers
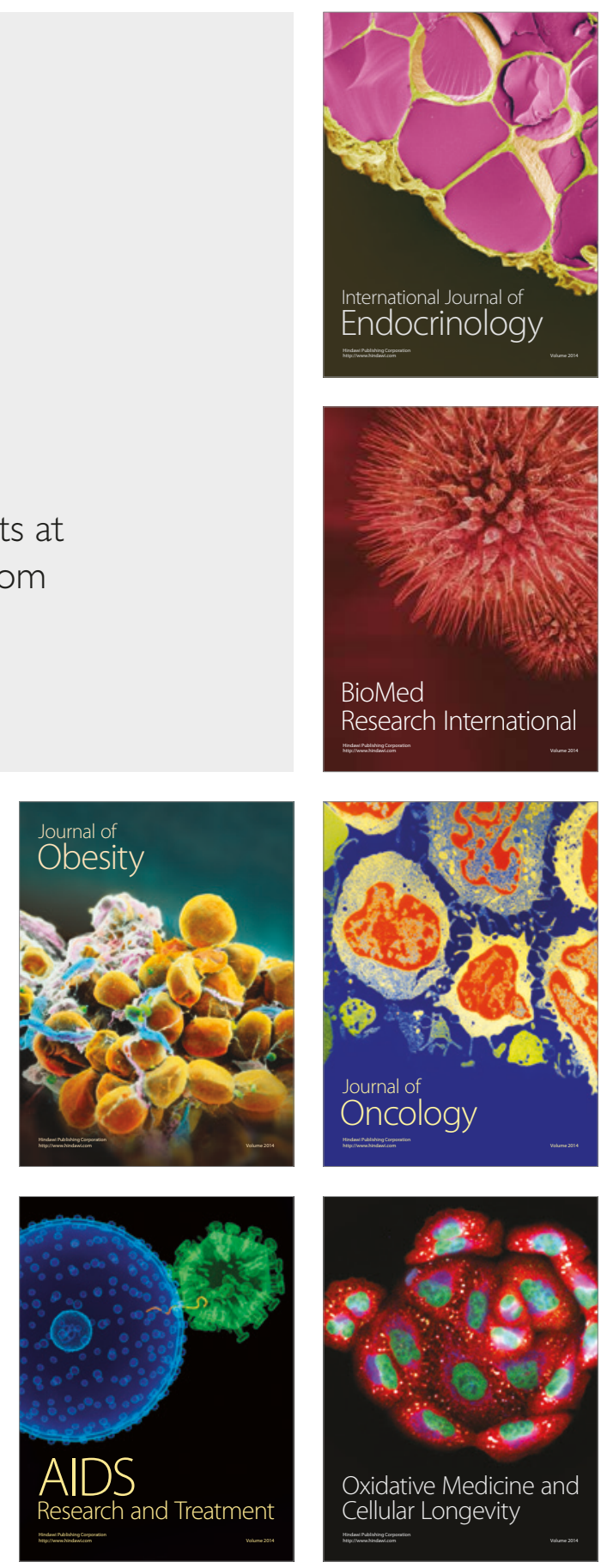